\title{
Mesenchymal stem cells attenuate acetic acid-induced colitis in rats via immunomodulatory effects
}

\author{
Hadeel A. Fawzy ${ }^{*}$, Mohamed F. El-yamany ${ }^{2}$ Hala M. Fawzy $^{1}$ and Ebtehal M. Fikry ${ }^{1}$ \\ ${ }^{1}$ Department of Pharmacology, National Organization for Drug Control and Research, NODCAR, Giza, Egypt \\ ${ }^{2}$ Department of Pharmacology\& Toxicology, Faculty of Pharmacy, Cairo University, Cairo, Egypt \\ *Correspondence: Haymanfawzy@ gmail.com, National Organization for Drug Control and Research, 6 Abou Hazem Steet, \\ El-Haram, Giza, 01142128989
}

\section{Article history: Received 05/06/2021}

Revised 13/08/2021

Accepted 16/08/2021

\begin{abstract}
Mesenchymal stem cells (MSCs) have recently gained popularity as a treatment for a variety of diseases. In this work, bone marrow MSCs (BM-MSCs) and adipose MSCs (AD-MSCs) were compared to dexamethasone (Dex) in the management of colitis induced by acetic acid in rats. Rats were divided in a random way into five groups: (1) Control, (2) Acetic acid treated (2 $\mathrm{ml}$ of $3 \%$ of acetic acid solution, intra-rectally as a single dose), (3) BM-MSCs treated (single i.p. administration of $1 \times 10^{6}$ cells/rat, 24 hours after acetic acid exposure), (4) AD-MSCs treated (single i.p. administration of $1 \times 10^{6}$ cells/ rat, 24 hours after acetic acid exposure), and (5) Dex treated $(0.5 \mathrm{mg} / \mathrm{kg} /$, i.p., 1 hour after acetic acid exposure) for 7 days. Following that, after 15 days of colitis induction, all rats were decapitated. Acetic acid induced colitis was marked by elevation of edema marker, myeloperoxidase, malondialdehyde, interleukin-1 $\beta$, tumor necrosis factoralpha,interleukin-6 apoptosis via caspase-3 expression, fibrosis using Masson's trichrome stain as well as infiltration of inflammatory cells by means of hematoxylin and eosin stain, It also lowered superoxide dismutase, total antioxidant, and interleukin-10 levels. After receiving either BM-MSCs or AD-MSCs, these adverse consequences were dramatically reduced. Finally, antioxidant, anti-inflammatory, antiapoptotic, and antifibrotic activities were detected in AD-MSCs, and BM-MSCs, indicating that they could be promising novel therapeutics for the treatment of colitis.
\end{abstract}

Keywords: Colitis; Mesenchymal stem cell; immunomodulation; inflammation; apoptosis, inflammation.

This is an open access article distributed under the CC BY-NC-ND license https://creativecommons.org/licenses/by/4.0/

\section{INTRODUCTION}

Ulcerative colitis and Crohn's diseases are two kinds of inflammatory bowel disease (IBD), which is a chronic and affects people all over the world. ${ }^{1}$. Colitis is a complex term referring to the inflammation of the inner lining of the colon ${ }^{2}$ owing to variety of reasons. The first cause is infection by certain microorganisms such as bacteria ${ }^{3}$, viruses ${ }^{4}$ or parasites ${ }^{5}$.This is called infectious colitis. The second cause is autoimmune disorders that include Crohn's diseaseandulcerative colitis ${ }^{6}$. The third cause is ischemia, allergic reactions ${ }^{7}$ or using some drugs like NSAIDs, mycophenolate, ipilimumab and retinoic acid ${ }^{8}$. Furthermore, colitis can happen because the colon wall becomes engorged with collagen or lymphocytes and this is called microscopic colitis as diagnosis can only be done by histological examination.
${ }^{9}$ Trinitrobenzene sulfonic acid (TNBS), dextran sodium sulfate (DSS), peptidoglycan, nonsteroidal anti-inflammatory medications, and acetic acid (Ac.a) have all been shown to cause colitis in experimental animals ${ }^{10,11}$. Ac.a induced colitis is the most commonly utilized and the easiest model to be applied ${ }^{12}$. Ac.a-induced colitis is a model of colitis that, in terms of etiology, histological characteristics, and inflammatory mediator profile, is very similar to human colitis ${ }^{10}$.

The pathogenesis of colitis is thought to be influenced by oxidative stress ${ }^{13}$, inflammatory mediators, immunology, and genetics ${ }^{14}$. Colitis is caused by a chronic intestinal inflammation that is exacerbated by acquired immune system cells ${ }^{15}$. Overactive lymphocytes and their pro-inflammatory cytokines may cause chronic inflammation by

Cite this article: Fawzy, ${ }_{\text {. }}$ El-Yamany, M. Fawzy, $H$., Fikry, E. Mesenchymal Stem Cells attenuate Acetic acid-Induced Colitis in Rats via Immunomodulatory Effects. Azhar International Journal of Pharmaceutical and Medical Sciences, 2022; 2(1): 96-107. doi: 10.21608/aijpms.2022.79293.1079 
overweighing the normal control mechanisms such as regulatory lymphocytes and anti-inflammatory cytokines such as transforming growth factor- $\beta$ (TGF- $\beta$ ) and interleukin-10 (IL-10) to reduce inflammation ${ }^{16}$. It can also be caused by $\mathrm{T}$ cells' resistance to undergo apoptosis after a certain activation $^{14}$.

Unspecialized cells that can differentiate into a range of specialized cells that make up the various tissues in our bodies are known as stem cells. ${ }^{17}$. They are known for their ability to self-renew through mitotic cell division. ${ }^{18}$. Injected mesenchymal stem cells (MSCs) are characterized by migrating to the bone marrow in the steady-state and then homing to the inflammation site by migrating across the endothelium and then entering the damaged organ, and they mediate immunomodulatory activities after injection because they have many chemokine receptors to chemo-attractants generated by the site of inflammation ${ }^{19}$.

Furthermore, MCSs can generate soluble factors that may play a greater role in tissue repair than their differentiation capacity, a phenomenon known as the paracrine effect. These soluble factors include, TGF1, nitric oxide, hepatocyte growth factor, prostaglandin-E2, IL-6, indoleamine 2, 3 dioxygenaseandheme oxygenase- $1^{5}$.

Bone marrow mesenchymal stem cells (BM-MSCs) are found in the stroma of the bone marrow in extremely modest numbers (about $0.001 \%-0.01 \%$ of total nucleated cells in the marrow $)^{20}$, When the same quantity of adipose tissue is separated, the number of adipose-derived stem cells (AD-MSCs) is approximately 500-fold higher ${ }^{21}$. Although both kinds have similar differentiation capacity, ADMSCs may have a higher proliferation rate ${ }^{22}$. In terms of immunophenotypes, they are nearly identical by more than $90 \%{ }^{23}$. Furthermore, BMMSCs show a higher proclivity for expressing angiogenic cytokines like insulin-like growth factor and vascular endothelial growth factor ${ }^{22}$.

The objective of this work was to see how effective BM-MSCs and AD-MSCs are at treating acetic acidinduced colitis compared to Dex, which was chosen as the reference drug because of its antiinflammatory and immunomodulatory properties ${ }^{24}$.

\section{METHODS}

\subsection{Animals}

In this investigation, adult Wistar male albino rats, 3:4 months old, balancing 180-230 g were employed. They were gotten from the animal stock of the National Organization for Drug Control and Research (NODCAR, Cairo, Egypt).
Animals were housed in the lab room for one week preceding to testing. They were housed under regular living settings, with room temperatures set at 23$27^{\circ} \mathrm{C}$ and humidity set at 50-70\%, with 12 hour luminance and gloom cycles, and free access to food, standard pellet diet, and water was permitted $a d$ libitum. Everything in the animal techniques was done in accordance with the Ethics Committee of the Faculty of Pharmacy Cairo University (permit number: PT-1944) and in accordance with the National Institutes of Health's Guide for the Care and Use of Laboratory Animals (NIH Publication No. 8523, revised 1996). Animals were not disturbed unnecessarily, and there was no pressure or difficult maneuvering.

\subsection{Experimental design}

Rats were haphazardly allocated into five groups ( $n=10$ per each group) as follows: Group 1(control) served as normal control, received intra-rectal normal saline once and then intra-peritoneal for 7 days. Group 2 (Ac.a) served as positive control, rats treated with a single dose of $2 \mathrm{ml}$ of $3 \%$ acetic acid, intra-rectally. Group 3 (Ac.a +BM-MSCs) and group 4 (Ac.a+AD-MSCs) where rats were treated with a single dose of $2 \mathrm{ml} \mathrm{3 \%}$ acetic acid, intra-rectally followed after 24 hours by a single intraperitoneal injection of $1 \times 106 \mathrm{BM}-\mathrm{MSCs}$ or $1 \times 106 \mathrm{AD}-\mathrm{MSCs}$ in $500 \mu \mathrm{l}$ phosphate buffer, respectively [25, 26]. Group 5 (Ac.a+Dex) rats were treated with a single dose of $2 \mathrm{ml} \mathrm{3 \%}$ acetic acid, intra-rectally, followed after 1 hour by Dex $(0.5 \mathrm{mg} / \mathrm{kg}$, i.p for 7 days) [27]. All rats were decapitated after 15 days from the beginning of the experiment.

At the end of the experiment, animals were decapitated under light anesthesia and the colon was isolated from each rat, and then the used animals were frozen till being incinerated. A part of the colon was homogenized in $10 \% \mathrm{w} / \mathrm{v}$ PBS, centrifuged at $4000 \mathrm{rpm} 4^{\circ} \mathrm{C}$ for $15 \mathrm{~min}$ and the supernatants were then refrigerated at $80^{\circ} \mathrm{C}$ to be tested for oxidative stress indicators and cytokines. Another portion was devoted to mRNA isolation for Real-Time Polymerase Chain Reaction (RT-PCR) detection and quantification of IL-1 $\beta$ mRNA. A section of the colon, three rats per group, was preserved in $10 \%$ formalin-saline and subjected to histological investigation using Masson's trichrome stain for fibrosis percentage recognition, hematoxylin and eosin (H\&E) stain for the detection of inflammatory scores or staining with Prussian blue to detect the MSCs homing in the colon tissue. It was also used for immunohistochemical analysis for the detection of caspase-3. 


\subsubsection{BM-MSCs isolation}

Bone marrow was extracted from male albino rats, 6 weeks old femurs by flushing them with Dulbecco's modified Eagle's medium enhanced with $10 \%$ fetal bovine medium. After that, cells with nuclei were segregated according to the density gradient and re-suspended in complete culture medium with $1 \%$ penicillin-streptomycin. The cells were maintained at $37^{\circ} \mathrm{C}$ in $5 \%$ humidified $\mathrm{CO} 2$ for 12-14 days for the production of large colonies,. The cultures were rinsed twice in PBS before being trypsinized for 5 minutes at $37^{\circ} \mathrm{C}$ with 0.25 percent trypsinin $1 \mathrm{mM}$ EDTA and centrifuged. The cells were suspended in a serum-enhanced media and incubated in a $50 \mathrm{~cm} 2$ culture flask $(2400 \mathrm{rpm}, 20$ min) after centrifugation. "Second passage cultures" were coined to describe the cultures that developed 28.

\subsubsection{AD-MSCs isolation}

Adipose tissue from the fat pad in the inguinal area in a completely sterile environment was obtained, and the tissue was then subjected to 60 minutes of enzymatic digestion in Hank's Balanced Salt Solution with 0.075 percent collagenase II at $37^{\circ} \mathrm{C}$, before being stopped with DMEM/F12 medium. After a 24-hour attachment period, the cells that were non-adherent were eliminated by rinsing in PBS. Devoted cells were grown in vitro in DMEM/F12 medium complemented with $10 \%$ fetal bovine serum, 0.1M Dex, streptomycin, and 1.25 $\mathrm{mg} / \mathrm{L}$ amphotericin B until passage two.

The adhesiveness and fusiform shape of both types of MSCs were seen in the culture. MSC surface markers CD90, CD271, and CD73 were determined using flow cytometry. Both AD-MSCs and BMMSCs had their cells counted using a hemocytometer 29 .

2.2.3 Labeling of mesenchymal stem cells by superparamagnetic iron oxide

$1 \mu \mathrm{l}$ of super magnetic iron oxide was added to 2 $\mathrm{ml}$ of culture medium and thoroughly mixed to attain a final concentration of $14 \mu \mathrm{g} / \mathrm{ml}$. After digestion and centrifugation, MSCs of the sixth generation were mixed thoroughly in the aforementioned solution and cultured for 24 hours at $37^{\circ} \mathrm{C}$ in a humidified incubator with $5 \% \mathrm{CO} 2$. After that, the labeled stem cells' colon tissue is excised and fixed on a slide for Prussian blue staining and examination under an electron microscope. Prussian blue staining revealed intracytoplasmic blue-stained positive particles under a transmission electron microscope $\mathrm{e}^{30}$.

\subsubsection{Evaluated parameters in the colon tissue}

\subsubsection{Edema marker}

The weight and length of each colon were measured, and the edema marker was determined using the equation below.

Edema marker $=$ colon weight $(\mathrm{g}) \times$ colon length $(\mathrm{cm})$ $\mathrm{x} 100^{31}$.

\subsubsection{Macroscopic examination}

The severity of macroscopically evident colonic injury was graded on a 0-3 scale using the scoring methodology. This approach considers the absence or presence of hyperemia, necrosis, and ulcers ${ }^{32}$.

2.2.4.3 Biochemical measurements in the colon homogenate

\subsection{Oxidative stress biomarkers}

The superoxide dismutase (SOD) activity and the total antioxidant capacity (TAC) in the colon were tested using reagent kits, as directed by the manufacturer (Biodiagnostic Company, Giza, Egypt). Myeloperoxidase (MPO) activity and malondialdehyde content (MDA) were measured biochemically, on the other handaccording to $^{33 \text {, }}$ ${ }^{34}$ (Bradley et al. (1982), Buege and Aust (1978)), respectively.

\subsection{Cytokines determination}

ELISA rat kits given by Cusabio kit (China) were used to measure, IL-10, tumor necrosis factor-alpha (TNF-) and IL-6 in the colon homogenate.

\subsection{IL-1 $\beta$ expression by quantitative real-time PCR (RT-PCR)}

The purity of the RNA was validated by spectrophotometric methods at $260 \mathrm{~nm}$ after total RNA was removed from tissue of the colon using the SV Total RNA method of isolation. The RNA was then reverse-transcribed into cDNA using a stratagene RT-PCR kit (Santa Clara, CA) according to the manufacturer's advices. To consider the expression of the IL-1 $\beta$ gene, quantitative real-time PCR was done using SYBR Green PCR Mastermix (Qiagen, Germany) according to the manufacture's protocol. In a final reaction volume of $50 \mu \mathrm{L}, 25 \mu \mathrm{L}$ of QuntiFast SYBR Green PCR Master Mix, $2 \mu \mathrm{L}$ primer pair mix (5 pmol/ $\mu$ Leach primer), $0.5 \mathrm{LcDNA}$, and $22.5 \mathrm{LdH} 2 \mathrm{O}$ were combined. The primer sequences for the IL- $1 \beta$ gene and the $\beta$-actin which is the housekeeping gene are listed in Table 1 . 
The AmpliTaq DNA polymerase activity was initiated with 10 minutes at $95{ }^{\circ} \mathrm{C}$, followed by 40 cycles of $95{ }^{\circ} \mathrm{C}$ for 15 seconds for denaturation and $60{ }^{\circ} \mathrm{C}$ for 1 minute for extension and annealing. The difference in the $\Delta \mathrm{Ct}$ values between the experimental and control samples $\Delta \Delta \mathrm{Ct}$ is calculated. The fold-change in expression of the IL-1 $\beta$ gene between the two samples is then equal to $2^{\wedge}(-\Delta \Delta \mathrm{Ct})$. The $\Delta \mathrm{Ct}$ was evaluated via taking $\beta$-actin $\mathrm{Ct}$ from that of IL-1 $\beta$ gene whereas $\Delta \Delta \mathrm{Ct}$ was achieved by taking the $\Delta \mathrm{Ct}$ of the standard sample (internal control) from that of the test sample. The relative expression ratios were determined by $2-\Delta \Delta \mathrm{ct}^{35}$.

\subsection{Statistical analysis}

The data was presented in the form of means \pm SEM. The Tukey-Kramer multiple comparisons test was done to compare means after using one-way ANOVA. For statistical analysis, the Graph Pad Prism software (version 5) was utilized; statistical significance was defined as a probability level of less than 0.05 .

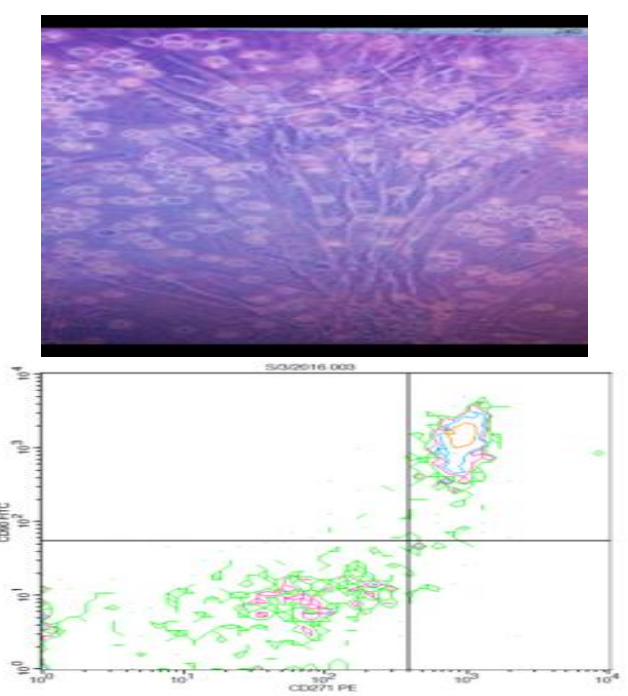

\section{RESULTS}

\subsection{MSCs identification}

Isolated undifferentiated MSCs showed typical adherent spindle and fibroblast-like shape and delivered $70-80 \%$ confluence after 14 days of culture. MSCs showed positive CD90, CD271 and CD73 with negative CD34 figure by flow cytometric analysis (figure (1)) and this assures that these cells were already MSCs.

\subsection{Homing of MSCs into the colon tissue}

Homing of MSCs into the colon tissue was verified by the detection of intra-cytoplasmic bluestained positive particles after Prussian blue staining under a transmission electron microscope (figure $(2 \mathrm{~A}, \mathrm{~B}))$ and this confirms that MSCs have reached the colon tissue.

\subsection{Antiedematous effect of MSCS}

BM-MSCs, AD-MSCs, and Dex decreased edema marker significantly by $34 \%, 27 \%$ and $35 \%$, respectively, as compared to Ac.a group (figure (3)), so MSCs have an antioedematous effect.

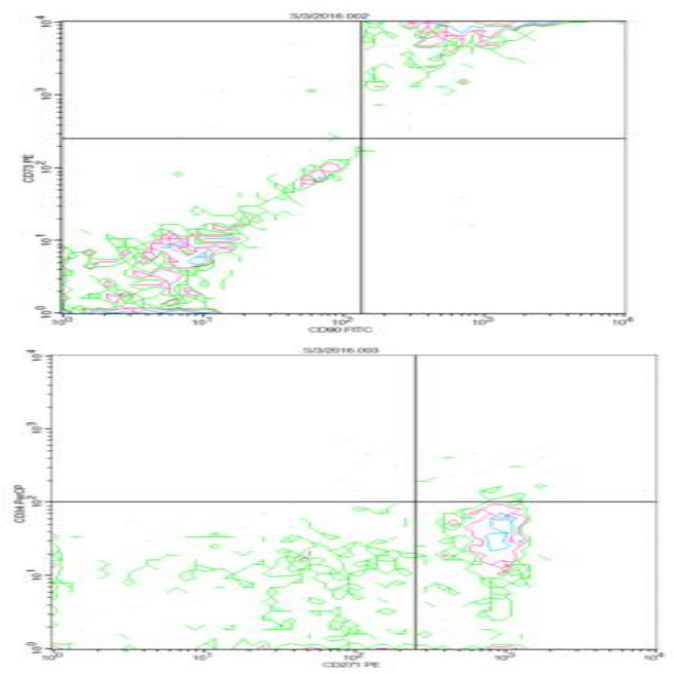

Figure 1. Typical morphological aspects of MSCs where they were identified by their fusiform fibroblastlike structure and flow cytometric characterization analysis; showing cells that were uniformly positive for CD90, and CD271 and CD73 with negative CD34 (x40). 


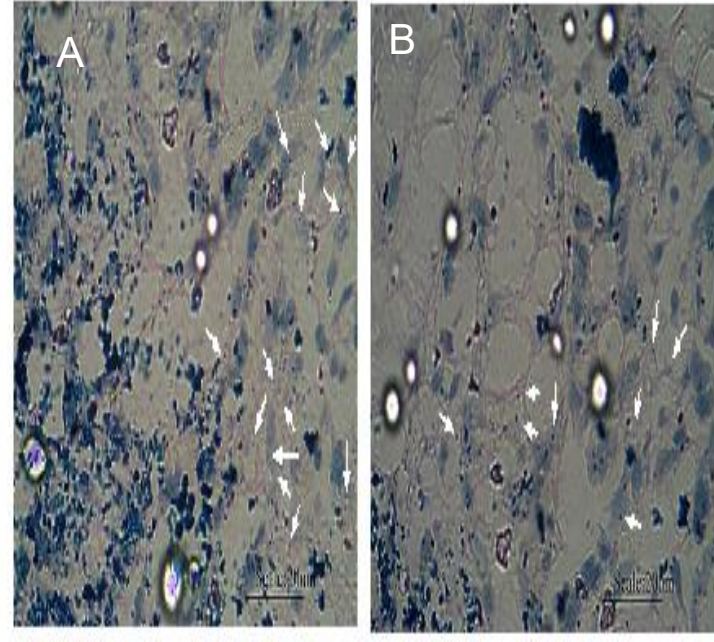

Figure 2. A: Homing of BM- MSCs and B: AD-MSCs into the rat colon $(\mathrm{x} 1000)$

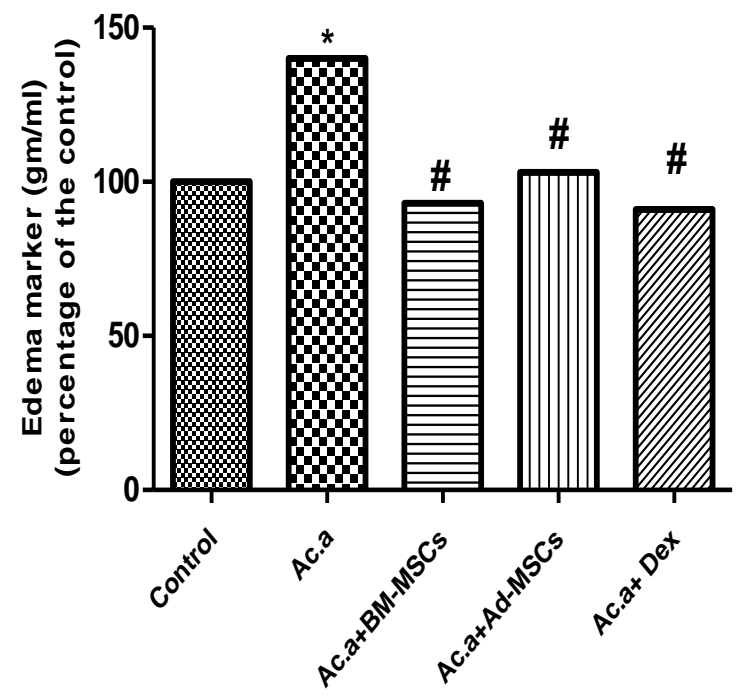

Figure 3. Effect of treatment with BM-MSCs or ADMSCs on edema marker compared to dexamethasone cells, AD-MSCs; adipose mesenchymal stem cells, Ac.a; acetic acid, Dex; dexamethasone.

\subsection{Effect of AD-MSCs or BM-MSCs on macroscopic examination scores (congestion, ulcers, and bleeding)}

By comparing BM-MSCs, AD-MSCs and Dex groups with Ac.a group, there was a significant remarkable decrease in the scores by a ratio of $81 \%$, $91 \%$, and $72 \%$, respectively (figure (4)) and as a result, MSCs can protect against congestion, ulcers, and bleeding.

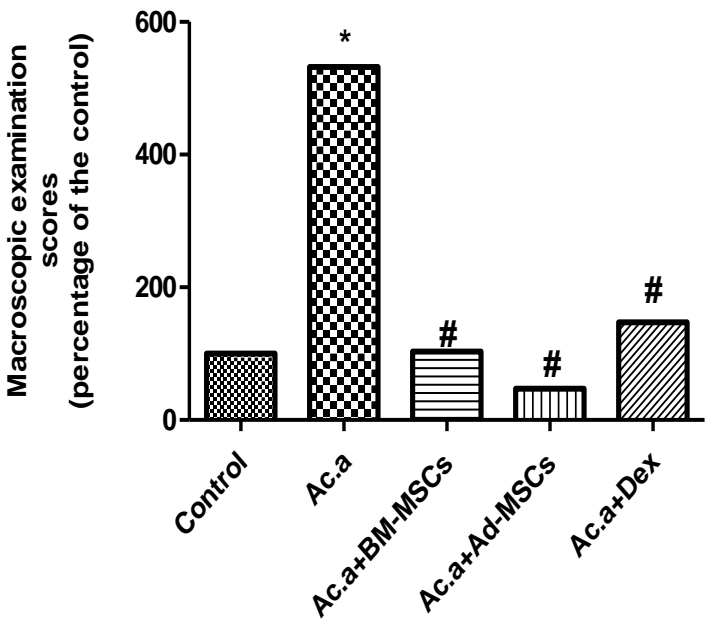

Figure 4. Effect of BM-MSCs or AD-MSCs on macroscopic examination scores (congestion, ulcers and bleeding)

Each value represents the percentage of 6-8 animals. Data were analysed by one-way ANOVA followed by TukeyKramer multiple comparisons test. ${ }^{*}<0.05$ versus the control group, $" \mathrm{p}<0.05$ versus Ac.a group. Where, BMMSCs; bone marrow mesenchymal stem cells, AD-MSCs; adipose mesenchymal stem cells, Ac.a; acetic acid, Dex; dexamethasone.

\subsection{The impact of MSCs on indicators of oxidative stress}

Ac.a injection induced a significant rise in colon peroxides represented as MPO that elevated to $16 \%$ and MDA that rose to $300 \%$ as compared to the control group. Furthermore, it caused a significant decrease in the antioxidant activity as total antioxidant lessened to $18 \%$ and SOD declined to $21 \%$ compared to the control group. Administration of AD-MSCs or BM-MSCs improved these deleterious properties significantly where they normalized the MPO activity as compared to the control group. BM-MSCs, AD-MSCs, and Dex diminished the MDA contents by $68 \%, 62 \%$, and $71 \%$, respectively compared to the Ac.a group. Instead, both AD-MSCs and BM-MSCs raised the colon content of TA to $21 \%$ and pushed up SOD activity to $34 \%$ and $28 \%$, respectively, as compared to Ac.a treated group whereas, Dex raised the SOD activity by only $17 \%$ as compared to the Ac.a group. The previous results emphasize that MSCs have antioxidant activity.

The effects of MSCs on oxidative stress indicators are shown in table 2 . 
Table 1. The sequences of primers of IL-1 $\beta$ gene and housekeeping gene ( $\beta$-actin).

\begin{tabular}{|c|l|}
\hline \multicolumn{2}{|c|}{ Primer sequence (5'-3') } \\
\hline IL-1 $\beta$ & $\begin{array}{l}\text { Forward: 5'- TGA TGT CCC ATT AGA CAG C -3' } \\
\text { Reverse: 5'-GAG GTG CTG ATG TAC CAG TT-3' }\end{array}$ \\
\hline$\beta$ - actin & $\begin{array}{l}\text { Forward : 5'-GGTCGGTGTGAACGGATTTGG-3' } \\
\text { Reverse:5'- ATGTAGGCCATGAGGTCCACC-3' }\end{array}$ \\
\hline
\end{tabular}

Table2. Effects of BM-MSCs and AD-MSCs on oxidative stress biomarkers compared to Dex in rats subjected toAc.a

\begin{tabular}{|c|c|c|c|c|}
\hline \multicolumn{5}{|c|}{ Parameters } \\
\hline Groups & $\begin{array}{c}\text { MPO } \\
\text { (U/mg protein) }\end{array}$ & $\begin{array}{c}\text { MDA } \\
\text { (nmol/gm tissue) }\end{array}$ & $\begin{array}{c}\text { TAC } \\
(\mathbf{m M} / \mathbf{m g} \\
\text { protein })\end{array}$ & $\begin{array}{c}\text { SOD } \\
\text { (u/gm tissue) }\end{array}$ \\
\hline $\begin{array}{l}\text { Control } \\
\text { Ac.a } \\
\text { Ac.a+BM-MSCs } \\
\text { AC.a+AD-MSCs } \\
\text { Dex }\end{array}$ & $\begin{array}{l}2.30 \pm 0.0474 \\
2.66 \pm 0.0534^{*} \\
2.49 \pm 0.0251 \\
2.26 \pm 0.0429^{\#} @ \\
2.63 \pm 0.0591^{*}\end{array}$ & $\begin{array}{l}350 \pm 29.7 \\
932 \pm 93.4^{*} \\
302 \pm 28.1^{\#} \\
357 \pm 31.3^{\#} \\
275 \pm 15.8^{\#}\end{array}$ & 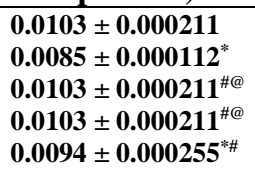 & $\begin{array}{l}1.31 \pm 0.0181 \\
1.03 \pm 0.0366^{*} \\
1.38 \pm 0.0508^{\# @} \\
1.32 \pm 0.0368^{\#} \\
1.21 \pm 0.0254^{\#}\end{array}$ \\
\hline
\end{tabular}

Values are expressed as mean \pm SEM of 6-8 rats per group. *vs. control group, \#vs. Ac.a group, @vs. Ac.a + Dex group (one-way ANOVA followed by Tukey-Kramer multiple comparisons test; $\mathrm{p}<0.05$ ). Where, BM-MSCs; bone marrow mesenchymal stem cells, AD-MSCs; adipose-derived mesenchymal stem cells, Ac.a; acetic acid, Dex; dexamethasone

Table 3. Effects of MSCs on Proinflammatory Cytokines

\begin{tabular}{|c|c|c|c|c|}
\hline \multicolumn{5}{|c|}{ Parameters } \\
\hline Groups & IL-1 & $\begin{array}{c}\text { TNF- } \alpha \\
(\text { Pg/mg protein })\end{array}$ & $\begin{array}{c}\text { IL-6 } \\
\text { (Pg/mg protein) }\end{array}$ & $\begin{array}{c}\text { IL-10 } \\
\text { (Pg/mg protein) }\end{array}$ \\
\hline $\begin{array}{l}\text { Control } \\
\text { Ac.a } \\
\text { Ac.a+BM-MSCs } \\
\text { AC.a+AD-MSCs } \\
\text { Dex }\end{array}$ & $\begin{array}{l}1.0 \pm 1.1 \\
11.8 \pm 0.729^{*} \\
5.1 \pm 0.355^{* \#} \\
3.6 \pm 0.301^{* \#} \\
4.1 \pm 0.428^{* \#}\end{array}$ & $\begin{array}{l}5.6 \pm 0.315 \\
21.2 \pm 0.513^{*} \\
14.5 \pm 0.238^{* \# @} \\
11.6 \pm 0.256^{* \# @} \\
8.3 \pm 0.148^{* \#}\end{array}$ & $\begin{array}{l}3.23 \pm 0.105 \\
12.30 \pm 0.442^{*} \\
8.10 \pm 0.110^{* \# @} \\
6.07 \pm 0.208^{* \# @} \\
4.20 \pm 0.110^{\#}\end{array}$ & $\begin{array}{l}20.7 \pm 0.559 \\
6.5 \pm 0.219^{*} \\
10.8 \pm 0.203^{* \# @ ~} \\
13.7 \pm 0.292^{* \# @} \\
17.1 \pm 0.538^{* \#} \\
\end{array}$ \\
\hline
\end{tabular}

Values are expressed as mean \pm SEM of 6-8 rats per group. *vs. control group, \#vs. Ac.a group, @vs. Ac.a + Dex group (one-way ANOVA followed by Tukey-Kramer multiple comparisons test; $\mathrm{p}<0.05$ ). Where, BM-MSCs; $\begin{array}{ll}\text { group (one-way ANOVA followed by Tukey-Kramer multiple comparisons test; } p<0.05) \text {. Where, BM-MSCs; } & \text { ving } \\ \text { bone marrow mesenchymal stem cells, AD-MSCs; adipose-derived mesenchymal stem cells, Ac.a; acetic acid, } & \text { :d to } \\ \text { Dex; dexamethasone. } & \end{array}$

\subsection{Effects of MSCs on Pro-inflammatory Cytokines}

Intra-rectal injection of Ac.a caused a significant rise in IL- $1 \beta$, TNF- $\alpha$, and IL-6 contents that were $1200 \%, 400 \%$, and $400 \%$, respectively, and it also caused prominent mitigation inIL-10 content to $69 \%$ when compared to the control group. Administration ofAD-MSCs, BM-MSCS or Dex hampered these harmful effects. They reduced IL-1 $\beta$ significantly by $57 \%, 70 \%$, and $66 \%$, respectively. Also, BM-MSCs, AD-MSCs or Dex treatment declined TNF- $\alpha$ content significantly when the Ac.a group. Moreover, there was a significant decrease of IL-6 content in BM-MSCs, A D-MSCs and Dex groups to $34 \%, 51 \%$, and $66 \%$, respectively when compared to the Ac.a group. It also seemed that BM-MSCs, AD-MSCs, and Dex elevated the content of IL-10 significantly when compared with the Ac.a animals by $66 \%, 200 \%$, and $300 \%$, respectively, so MSCS have immunomodulatory and antiinflammatory activity.

Table 3 elucidates MSCs' impact on proinflammatory cytokines. 

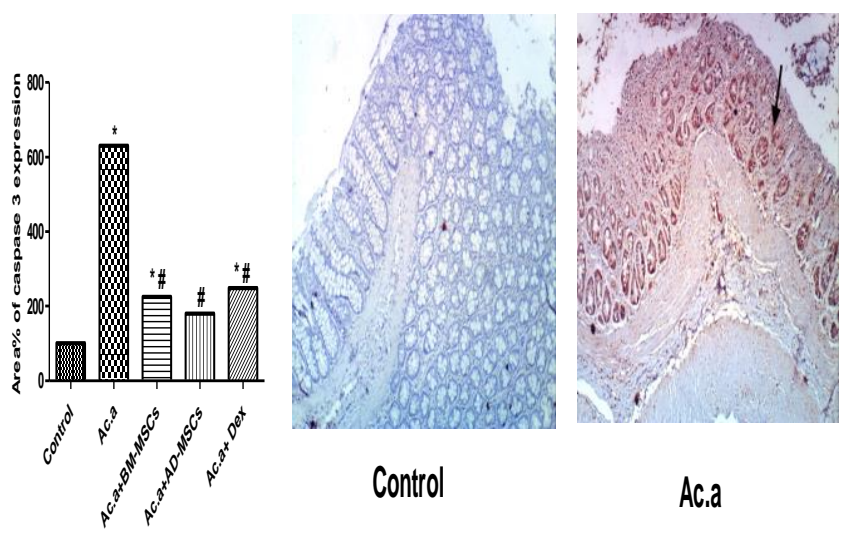

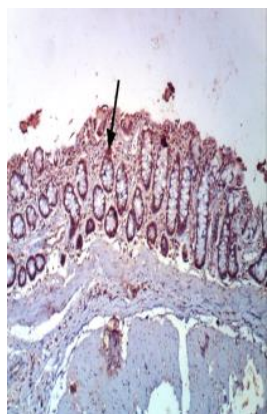

Ac.a+BM-MSCs

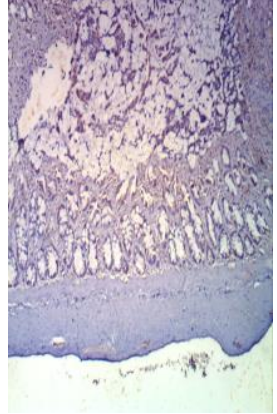

Ac.a+AD-MSCs

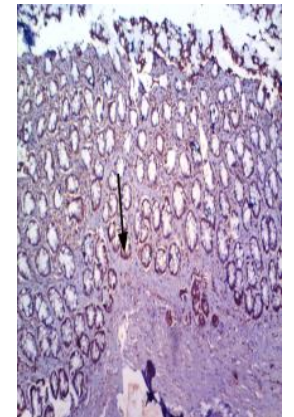

Dex
Figure 5. Effect of treatment with BM-MSCs or ADMSCs on the colon expression of caspase-3 compared to dexamethasone

Each bar represents the percentage of 15 sections ( 3 per group).Data was analysed by one-way ANOVA followed by Tukey-Kramer multiple comparisons test. ${ }^{*}<0.05$ versus the control group and ${ }^{\#} \mathrm{p}<0.05$ versus Ac.a group. Where, BM-MSCs; bone marrow mesenchymal stem cells, AD-MSCs; adipose mesenchymal stem cells, Ac.a; acetic acid and Dex; dexamethasone and photomicrographs of colon sections showing the effect of treatment with BMMSCs or AD-MSCs compared with dexamethasone on the colon expression of caspase- 3 in rats subjected to acetic acid as shown by microscopical examination by immunohistochemical assay (x100).

\subsection{Antifibrotic effect of MSCs}

Fibrosis was highly noticed in the Ac.a animals (200\%) as compared to the control group. Notably, administrations of AD-MSCs, BM-MSCs or Dex have normalized fibrosis percentage as compared to the control group, so the antifibrotic activity of MSCs is evident. This is shown in figure (6A) and (6B).
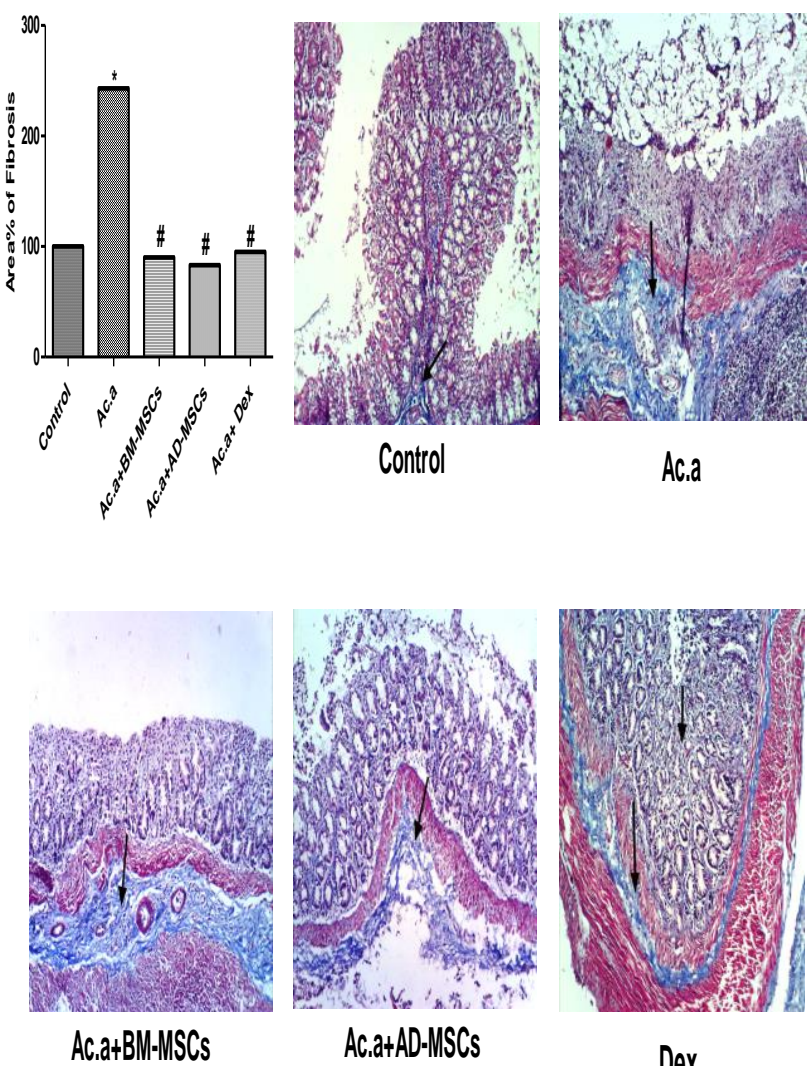

Dex

Figure 6. Effect of treatment with BM-MSCs or ADMSCs on fibrosis percentage compared to dexamethasone

Each bar represents the percentage of 15 sections ( 3 per group).Data was analysed by one-way ANOVA followed by Tukey-Kramer multiple comparisons test. ${ }^{*}<<0.05$ versus the control group and ${ }^{\#} \mathrm{p}<0.05$ versus Ac.a group. Where, BM-MSCs; bone marrow mesenchymal stem cells, AD-MSCs; adipose mesenchymal stem cells, Ac.a; acetic acid and Dex; dexamethasone and Photomicrographs of colon sections showing the effect of treatment with BMMSCs or AD-MSCs as compared with dexamethasone on fibrosis in rats subjected to acetic acid as shown by microscopical examination by using Masson's Trichrome (x100). Where, BM-MSCs; bone marrow mesenchymal stem cells, AD-MSCs; adipose mesenchymal stem cells, Ac.a; acetic acid, Dex; dexamethasone.

\subsection{Effect of MSCS on colon inflammation by histopathological examination using hematoxylin and eosin}

Administration of Ac.a triggered coagulative necrosis of the mucosal layer accompanied by submucosal edema, inflammatory cells infiltration and intraluminal accumulation of inflammatory exudate. Instead, administration of AD-MSCs, BMMSCs or Dex was accompanied by few inflammatory cell infiltration and fine strands of proliferation in the mucosa that has no significant difference when compared to the control group and 
these results ascertain that MSCs can protect against inflammation. This is elucidated in figure (7).
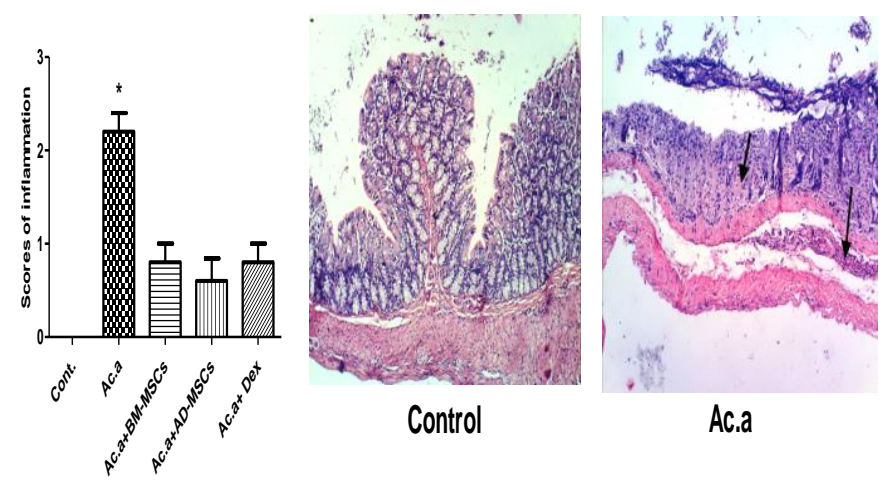

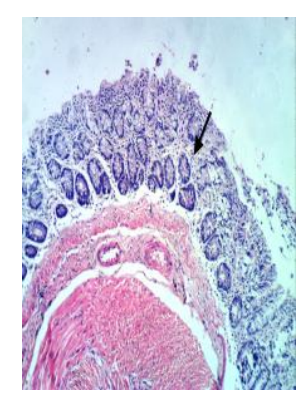

Ac.a+BM-MSCs

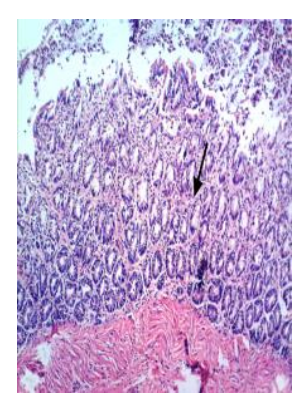

Ac.a+AD-MSCs

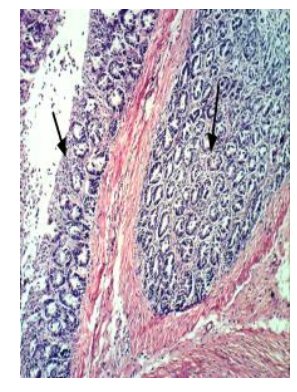

Dex
Figure 7. Effect of treatment with BM-MSCs or ADMSCs on colon inflammation compared to dexamethasone Each bar represents the median of 15 sections (3 per group) \pm range. Data was analyzed by Kruskal Wallis test followed by Dunn's multiple comparisons post test. ${ }^{*}<<0.05$ versus the control group. Where, BM-MSCs; bone marrow mesenchymal stem cells, AD-MSCs; adipose mesenchymal stem cells, Ac.a; acetic acid and Dex; dexamethasone and Photomicrographs of colon sections showing the effect of treatment with BM-MSCs or ADMSCs as compared with dexamethasone on colon inflammation in rats subjected to acetic acid as shown by microscopical examination by using hematoxyline and eosin stain (x100).

\section{DISCUSSION}

According to the authors' familiarity, this is the first study to compare two types of MSCs, AD-MSCs and BM-MSCs in the treatment of Ac.a triggered colitis to the corticosteroid Dex.

Intra-rectal injection of Ac.a led to a marked elevation in edema marker and macroscopic examination scores (congestion, ulcers, and bleeding). This goes in line with the previous studies ${ }^{36}$. Besides, Ac.a caused fibrosis which was detected by Masson's trichrome stain and it has also triggered inflammation, necrosis, ulceration, and edema that were detected by H\&E stain and this is in harmony with the previous study [37]. In contrast, MSCs administration stimulated colonic repair by decreasing fibrosis, necrosis, ulceration and edema. These results follow a previous study ${ }^{38}$. The antifibrotic and anti-inflammatory effects of MSCs could be explained by the ability of MSCs to induce colonic epithelial cell hyperplasia ${ }^{39}$, their differentiation character and paracrine activity ${ }^{40}$.

Herein, it was reported that Ac.a induced oxidative stress revealed by significant reductions in the TAC content and SOD activity. Besides, it induced significant elevations in MPO activity and MDA content. These conclusions are in an argument with the preceding studies 41,44 . Otherwise, the present study showed that BM-MSCs and AD-MSCs have a marked antioxidant activity. Herein, the TAC content and SOD activity were replenished, while MDA content and MPO activity were hampered as compared to the Ac.a group. These antioxidant properties of MSCs were in harmony with the previous studies ${ }^{45}$, but according to da Costa et al, ${ }^{46}$ who used AD-MSCs, MDA content didn't decrease significantly. According to Kemp et al, (2010), ${ }^{47}$ the MSCs' antioxidant activity may be owing to their ability to generate and release SOD, which is produced synergistically in response to TNF and interferon (IFN- $\gamma$ ). Furthermore, MSCs are a source of cysteine, which is consumed in GSH production, inhibiting $\mathrm{GSH}$ reduction in the affected colon progressively ${ }^{48}$.

In this colitis model, administration of Ac.a resulted in a significant increment in TNF- $\alpha$, IL-6, and IL- $1 \beta$ contents. As well as, it caused a significant decline in IL-10 content. These findings go along with the earlier studies ${ }^{49,50,51}$. Worth mentioning, MSCs caused an apparent depression in IL-1 $\beta$, TNF$\alpha$, and IL-6 contents. Also, MSCs groups had a significant rise in IL-10 as compared to the Ac.a treated group. This is in harmony with the previous studies, where colitis was chemically induced by TNBS administrated by enema ${ }^{52}$. This improvement may be attributed to stem cells characteristics. Injected MSCs can travel to the BM in the steadystate and home to the inflamed site across the endothelium and then the affected organ ${ }^{53}$.and mediate immunomodulatory actions after injection ${ }^{19}$. Also, MSCs can synthesize and release SOD in a synergistic way in response to inflammatory mediators TNF- $\alpha$ and IFN- $\gamma{ }^{47}$. Moreover, MSCs paracrine activity plays a key role in the regulation of hematopoiesis, angiogenesis, immune and inflammatory reaction ${ }^{54}$. Additionally, MSCs have the ability to influence the majority of immune system cells, influencing cellular proliferation, differentiation, maturation, and function ${ }^{55}$.

Furthermore, immunohistochemical detection in the current work revealed extensive expression of caspase- 3 in Ac.a treated group as compared to the 
control animals. This is in harmony with the previous studies 43,55,56 The mechanism of Ac.a induced caspase- 3 elevation can be described by Kaushal et $a l,(2001),{ }^{57}$ who stated that binding of TNF- $\alpha$ to its receptors activates receptor-dependent apoptotic pathways through activation of many caspases containing caspase- $3^{58}$. On the contrary, in In this work, BM-MSCs prevented apoptosis and ADMSCs protected rats from elevated caspase-3 expression, which is similar to previous research ${ }^{59}$. It is supposed that the mechanism by which apoptosis has improved is by decreasing the Bax to $\mathrm{Bcl}-2$ ratio and inhibit the activation of caspase-3, thus preventing colon apoptosis like in another study cared with myocardial infarction ${ }^{60}$ and proceeding cell cycle ${ }^{38}$.

\section{CONCLUSIONS}

Because of their immunomodulatory, antiinflammatory, antioxidant, and antiapoptotic properties, BM-MSCs and AD-MSCs are promising candidates for the therapy of colitis.

Current and future development: Application of MSCs therapy in the patients with ulcerative colitis.

Acknowledgment : Authors are grateful to DR. Hala Gabr for her efforts in isolation and labelling of stem cells. Moreover, the authors show appreciation to Dr. Kawkab Abdel-Aziz for her supporting role in the analysis of histopathology.

Conflict of interest: No conflict of interest declared by the authors.

Ethical approval statement: Everything in animals' techniques was done according to the faculty of Pharmacy Cairo UniversityEthics Committee (permit number: PT-1944) and meet the terms of the Guide for Care and Use of Laboratory Animals issued by the US National Institutes of Health (NIH Publication No. 85-23, revised 1996). Unnecessary disturbance of animals, pressure and tough maneuver were avoided.

Authors' contributions: All authors were hand by hand in each part of this research.

Funding statement: This research hasn't received any fund from anywhere.

\section{References}

1. Kaplan, G.G., The global burden of IBD: from 2015 to 2025. Nature reviews Gastroenterology \& hepatology, 2015. 12(12): p. 720-727.

2. Feuerstein JD, Cheifetz AS. Ulcerative colitis: epidemiology, diagnosis, and management. InMayo Clinic Proceedings 2014 Nov 89(11): p. (1553-1563). Elsevier.

3. Newton, J.M. and C.M. Surawicz, Infectious gastroenteritis and colitis, in Diarrhea. 2010, Springer. p. 33-59.

4. Kim, C.H., et al., Cytomegalovirus colitis in patients without inflammatory bowel disease: a single center study. Scandinavian journal of gastroenterology, 2010. 45(11): p. 1295-1301.

5. Hechenbleikner, E.M. and J.A. McQuade, Parasitic colitis. Clinics in colon and rectal surgery, 2015. 28(02): p. 079-086.

6. Xavier, R. and D. Podolsky, Unravelling the pathogenesis of inflammatory bowel disease. Nature, 2007. 448(7152): p. 427-434.

7. Protocol, A., ABM clinical protocol\# 24: allergic proctocolitis in the exclusively breastfed infant. Breastfeeding Medicine, 2011. 6(6): p. 8283.

8. Villanacci, V. and M. Salemme, Drug-Induced Colitis, in Colitis. 2014, Springer. p. 165-184.

9. Chatelain, D., N. Mokrani, and J.-F. Fléjou. Microscopic colitis: collagenous colitis and lymphocytic colitis. in Annales de pathologie. 2007 27(6) p. 448-458.

10. Randhawa, P.K., et al., A review on chemicalinduced inflammatory bowel disease models in rodents. .KJPP, 2014. 18(4): p. 279-288.

11. Antoniou, E., et al., The TNBS-induced colitis animal model: an overview. Annals of medicine and surgery, 2016. 11: p. 9-15.

12. MacPherson, B. and C. Pfeiffer, Experimental production of diffuse colitis in rats. Digestion, 1978. 17(2): p. 135-150.

13. Rana, S., et al., Role of oxidative stress \& antioxidant defence in ulcerative colitis patients from north India.IJMR, 2014. 139(4): p. 568569. 
14. Kim, D.H. and J.H. Cheon, Pathogenesis of Inflammatory Bowel Disease and Recent Advances in Biologic Therapies. Immune network, 2017. 17(1): p. 25-40.

15. Neurath, M.F., Cytokines in inflammatory bowel disease. Nature reviews. Immunology, 2014. 14(5): p. 329-342.

16. Fuss, I.J., et al., The interrelated roles of TGF$\beta$ and $I L-10$ in the regulation of experimental colitis. The Journal of Immunology, 2002. 168(2): p. 900-908.

17. Kalra, K. and P. Tomar, Stem Cell: Basics, Classification and Applications. Am. J. Phytomdicine Clin. Ther, 2014: p. 919-930.

18. Drummond-Barbosa, D. and A.C. Spradling, Stem cells and their progeny respond to nutritional changes during Drosophila oogenesis. Developmental biology, 2001. 231(1): p. 265-278.

19. Li, M. and S. Ikehara, Bone-marrow-derived mesenchymal stem cells for organ repair. Stem cells international, 2013. 2013: p. 1-8.

20. Bernardo, M.E., F. Locatelli, and W.E. Fibbe, Mesenchymal stromal cells: a novel treatment modality for tissue repair. Annals of the New York Academy of Sciences, 2009. 1176(1): p. 101-117.

21.Hass, R., et al., Different populations and sources of human mesenchymal stem cells (MSC): a comparison of adult and neonatal tissue-derived MSC. Cell Communication and Signaling, 2011. 9(1): p. 1-14.

22.Peng, L., et al., Comparative analysis of mesenchymal stem cells from bone marrow, cartilage, and adipose tissue. Stem cells and development, 2008. 17(4): p. 761-774.

23. Strioga, M., et al., Same or not the same? Comparison of adipose tissue-derived versus bone marrow-derived mesenchymal stem and stromal cells. Stem cells and development, 2012. 21(14): p. 2724-2752.

24. Taylor, K. and P.R. Gibson, Conventional Therapy of Ulcerative Colitis: Corticosteroids, in Crohn's Disease and Ulcerative Colitis. 2017, Springer. p. 399-412.
25. Robinson, A.M., et al., The neuroprotective effects of human bone marrow mesenchymal stem cells are dose-dependent in TNBS colitis. Stem Cell Research \& Therapy, 2017. 8(1): p. 87.

26. Soontararak, S., et al., Mesenchymal Stem Cells (MSC) Derived from Induced Pluripotent Stem Cells (iPSC) Equivalent to Adipose-Derived MSC in Promoting Intestinal Healing and Microbiome Normalization in Mouse Inflammatory Bowel Disease Model. Stem cells translational medicine, 2018. 7(6): p. 456-467.

27. Fikry, E.M., et al., Bone Marrow and AdiposeDerived Mesenchymal Stem Cells Alleviate Methotrexate-Induced Pulmonary Fibrosis in Rat: Comparison with Dexamethasone.JBMT, 2015. 29(7): p. 321-329.

28. Alhadlaq, A. and J.J. Mao, Mesenchymal stem cells: isolation and therapeutics. Stem cells and development, 2004. 13(4): p. 436-448.

29. Dueñas F, Becerra V, Cortes Y, Vidal S, Sáenz L, Palomino J, De los Reyes M, Peralta OA. Hepatogenic and neurogenic differentiation of bone marrow mesenchymal stem cells from abattoir-derived bovine fetuses. BMC veterinary research. 2014 Dec;10(1):p.1-3..

30. Cheng, J.-1., et al., In vivo tracing of superparamagnetic iron oxide-labeled bone marrow mesenchymal stem cells transplanted for traumatic brain injury by susceptibility weighted imaging in a rat model. Chinese Journal of Traumatology (English Edition), 2010. 13(3): p. 173-177.

31. Soliman, G.A., et al., Protective effects of two Astragalus species on ulcerative colitis in rats. Tropical Journal of Pharmaceutical Research, 2016. 15(10): p. 2155-2163.

32. Menozzi, A., et al., Long-term study of TNBSinduced colitis in rats: focus on mast cells. Inflammation research, 2006. 55(10): p. 416-422.

33. Bradley, P.P., et al., Measurement of cutaneous inflammation: estimation of neutrophil content with an enzyme marker. Journal of Investigative Dermatology, 1982. 78(3): p. 206-209.

34. Buege, J.A. and S.D. Aust, [30] Microsomal lipid peroxidation, in Methods in enzymology. 1978, Elsevier. p. 302-310. 
35. Pfaffl, M.W., A new mathematical model for relative quantification in real-time $R T-P C R$. Nucleic acids research, 2001. 29(9): p. e45-e45.

36. Ashry, E.E., et al., Protective Effect of Ketamine against Acetic Acid-Induced Ulcerative Colitis in Rats. Pharmacology \& Pharmacy, 2016. 7(01): p. 9-12.

37.El-Galil, T.I.A., T.A. ElGhamrawy, and A.O. El Sadik, The Effect of N. Acetylcysteine and Ginger on Acetic Acid Induced Colitis in Adult Male Albino Rats: Histological, Immunohistochemical and Morphometric Study. Journal of Cytology \& Histology, 2015. 3(2): p. $1-2$.

38. Fawzy, S.A., R.K.E.-d. Abo-Elnou, and D.F.A.-E.-M. El, The possible role of mesenchymal stem cells therapy in the repair of experimentally induced colitis in male albino rats. International journal of stem cells, 2013. 6(2): p. 92-95.

39. Tanaka, H., et al., Myogenic lineage differentiated mesenchymal stem cells enhance recovery from dextran sulfate sodium-induced colitis in the rat. Journal of gastroenterology, 2011. 46(2): p. 143-152.

40. Hayashi, Y., et al., Topical implantation of mesenchymal stem cells has beneficial effects on healing of experimental colitis in rats. Journal of Pharmacology and Experimental Therapeutics, 2008. 326(2): p. 523-531.

41. Cetinkaya, A., et al., Effects of L-carnitine on oxidant/antioxidant status in acetic acid-induced colitis. Digestive diseases and sciences, 2006. 51(3): p. 488-494.

42.Hagar, H.H., et al., Ameliorative effect of pyrrolidinedithiocarbamate on acetic acidinduced colitis in rats. European journal of pharmacology, 2007. 554(1): p. 69-77.

43. Cagin, Y.F., et al., Effects of dexpanthenol on acetic acid-induced colitis in rats. Experimental and therapeutic medicine, 2016. 12(5): p. 29582964.

44. Tanideh, N., et al., Healing acceleration of acetic acid-induced colitis by marigold (Calendula officinalis) in male rats. Saudi journal of gastroenterology: official journal of the Saudi Gastroenterology Association, 2016. 22(1): p. 50-54.
45. Marcelino, M.Y., et al., Cell therapy in experimental model of inflammatory bowel disease. Journal of Coloproctology (Rio de Janeiro), 2015. 35(1): p. 20-27.

46. da Costa Gonçalves, F., et al., Antioxidant properties of mesenchymal stem cells against oxidative stress in a murine model of colitis. Biotechnology letters, 2017. 39(4): p. 613-622.

47. Kemp, K., et al., Mesenchymal stem cellsecreted superoxide dismutase promotes cerebellar neuronal survival. Journal of neurochemistry, 2010. 114(6): p. 1569-1580.

48. Ishii, T. and G.E. Mann, Redox status in mammalian cells and stem cells during culture in vitro: critical roles of Nrf2 and cystine transporter activity in the maintenance of redox balance. Redox biology, 2014. 2: p. 786-794.

49. Stucchi, A., et al., A new transcription factor that regulates TNF- $\alpha$ gene expression, LITAF, is increased in intestinal tissues from patients with $C D$ and $U C$. Inflammatory bowel diseases, 2006. 12(7): p. 581-587.

50. González, M.A., et al., Adipose-derived mesenchymal stem cells alleviate experimental colitis by inhibiting inflammatory and autoimmune responses. Gastroenterology, 2009. 136(3): p. 978-989.

51. Tahan, G., et al., Vitamin E has a dual effect of anti-inflammatory and antioxidant activities in acetic acid-induced ulcerative colitis in rats. Canadian journal of surgery, 2011. 54(5): p. 333 339.

52. Wang, L.-T., et al., Human mesenchymal stem cells (MSCs) for treatment towards immune-and inflammation-mediated diseases: review of current clinical trials. Journal of biomedical science, 2016. 23(1): p. 1-13.

53. Devine, S.M., et al., Mesenchymal stem cells are capable of homing to the bone marrow of non-human primates following systemic infusion. Experimental hematology, 2001. 29(2): p. 244255.

54. Chen, L., et al., Paracrine factors of mesenchymal stem cells recruit macrophages and endothelial lineage cells and enhance wound healing. PloS one, 2008. 3(4): p. e1886.

55. Beyth, S., et al., Human mesenchymal stem cells alter antigen-presenting cell maturation 
and induce T-cell unresponsiveness. Blood, 2005. 105(5): p. 2214-2219.

56. Azza, A.A., et al., Protective effect of cardamonin against acetic acid-induced ulcerative colitis in rats. Pharmacological Reports, 2017. 69(2): p. 268-275.

57. Kaushal, G.P., et al., Role and regulation of activation of caspases in cisplatin-induced injury to renal tubular epithelial cells. Kidney international, 2001. 60(5): p. 1726-1736.

58. Labib, D.A.A., O.G. Shaker, and L.O. Elfarouk, Protective effects of nebivolol on acetic acid-induced ulcerative colitis in rats. Kasr Al Ainy Medical Journal, 2016. 22(3): p. 99-100.

59. Gashmardi, N., et al., Impacts of Bone Marrow Stem Cells on Caspase-3 Levels after Spinal Cord Injury in Mice. Iranian journal of medical sciences, 2017. 42(6): p. 593-596.

60.He, A., et al., The antiapoptotic effect of mesenchymal stem cell transplantation on ischemic myocardium is enhanced by anoxic preconditioning. Canadian Journal of Cardiology, 2009. 25(6): p. 353358. 